\title{
Application of Physics Model in prediction of the Hellas National election results
}

\author{
M. P. Hanias ${ }^{1}$ and L. Magafas ${ }^{2}$, \\ ${ }^{\prime}$ Technological and Educational Institute of Chalkis, GR 34400, Evia, Chalkis, Hellas \\ ${ }^{2}$ Department of Electrical Engineering, Kavala Institute of Technology, St. Loukas 65404 Kavala, Hellas.
}

Received 11 September 2009; Revised 14 September 2009; Accepted 16 September 2009

\begin{abstract}
In this paper we use a new scientific field called "DemoscopoPhysics" based on chaos theory to predict the Hellenic National election results in the form of time series for Hellenic political parties, New Democracy (ND), Panhellenic Social-istic Movement (PASOK), Hellenic Communistic Party (KKE), Coalition of the Radical Left (SYRIZA) and (Popular Orthodox Rally) LAOS". Using the results of our previous article we reconstruct of the corresponding strange attractor for each political party achieved up to a 30 time steps out of sample prediction of the public survey. Also reducing the degrees of freedom to 4 we have capture the polarization of voters.
\end{abstract}

Keywords: DemoscopoPhysics, Chaos, Forecasting Model.

\section{Introduction}

In our previous work [1] we used a new scientific field called "DemoscopoPhysics" based on chaos theory, to predict the Hellenic Euro election results for New Democracy (ND), Panhellenic Socialistic Movement (PASOK), Hellenic Communistic Party (KKE), Coalition of the Radical Left (SYRIZA) and (Popular Orthodox Rally) LAOS political parties. At present work we use the same model to predict the Hellenic National election results. We have approached the prediction with two different ways. Taking into account the results of opinion polls we have done regression in intension to vote. These new data are the raw data now. If we had applied statistical methods to these data we would take static results with very short horizon forecasting. For this reason we apply dynamic methods based on chaos theory in order to show the hidden potential of each political party and make predictions with a time horizon of 30 days. In first case we used the same parameters of our previous article [1]. That means that the value of embedding dimension was 6 for prediction, i.e. the number of degrees of freedom was 6 . In second case we have decreased the degrees of freedom to 4 to capture the polarization of voters.

\section{Public Survey Time Series}

To construct the time series we have taken into account the assessment vote from public surveys in Hellas from 16-1-2007 to 6-092009 the estimation of the election behavior of the unclarified vote based on previous elections, they were come into question the possible election results for the whole period. The number of

* E-mail address: Imagafas@otenet.gr ISSN: 1791-2377 (C) 2009 Kavala Institute of Technology. All rights reserved. raw data is 48 for each political party, and each data is the average value of 4 polling companies with mean error $1.5 \%$ for ND and PASOK political parties and $1 \%$ for other political parties. In order to reconstruct the phase space from experimental data these data should be constituted by sampled points of equal time-distances. In our case this condition is not fulfilled. For this purpose we inter-polate our raw data with cubic spline so we have create $\mathrm{N}=1057$ points with a sample rate of 1 day.

\section{Time series prediction}

For a scalar time series, in our case the gallop poll time series, the phase space can be reconstructed using the methods of delays. The basic idea in the method of delays is that the evolution of any single variable of a system is determined by the other variables with which it interacts. Information about the relevant variables is thus implicitly contained in the history of any single variable. On the basis of this an "equivalent" phase space can be reconstructed by assigning an element of the time series series $\mathrm{x}_{\mathrm{i}}$ and its successive delays as coordinates of a new vector time series $\vec{X}$. The reconstructed $m$-dimensional signal projected into the state space can exhibit a range of trajectories, some of which have structures or patterns that can be used for system prediction and modeling [1]. We predict evolution of the percentages of votes for each political party, by computing weighted average of evolution of close neighbors of the predicted state in the reconstructed phase space [1-5]. We used the values of $\tau$ and $\mathrm{m}$ from our previous article [1]. Actual and predicted time series for $\mathrm{k}=30$ time steps ahead are presented at Figs 1.(a),(b),(c),(d),(e) for ND, PASOK, KKE, SYRIZA, LAOS respectively. 


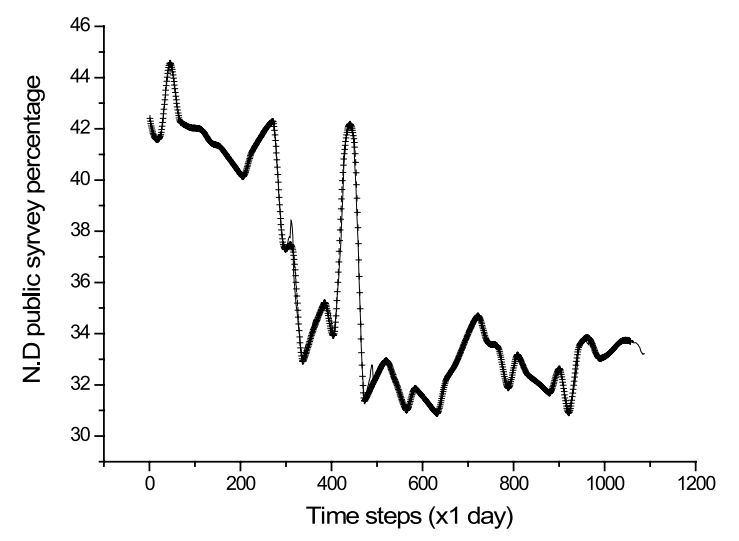

(a)

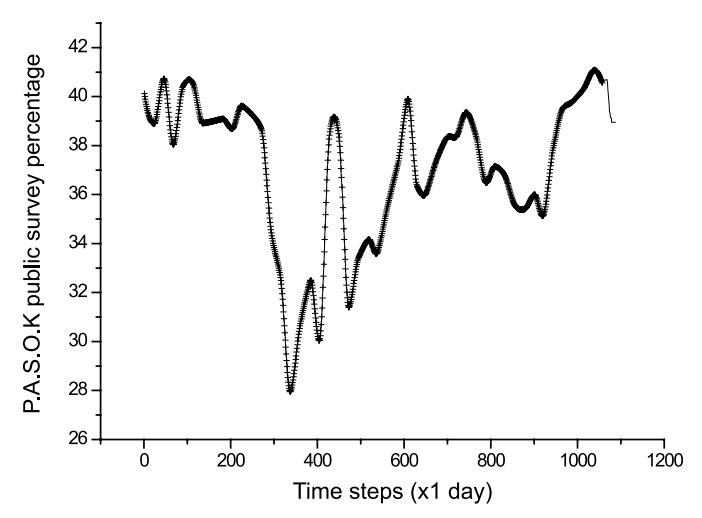

(b)

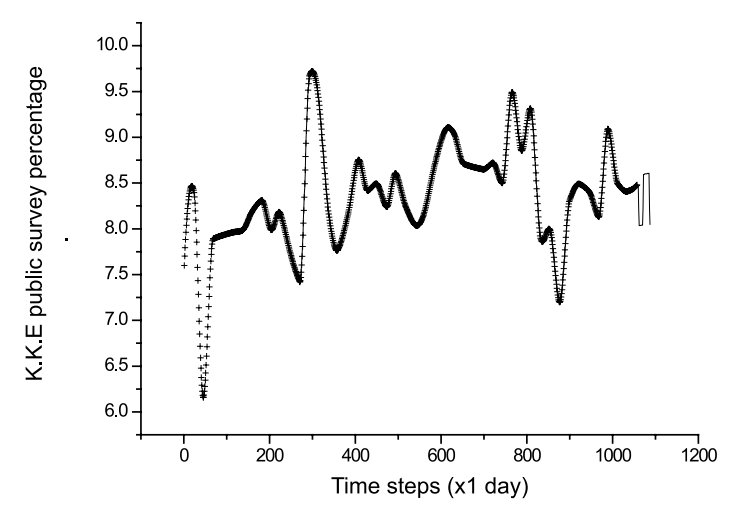

(c)

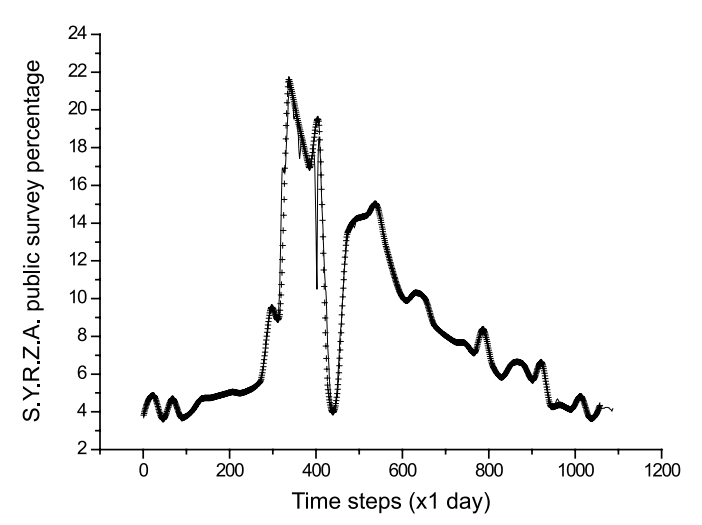

(d)

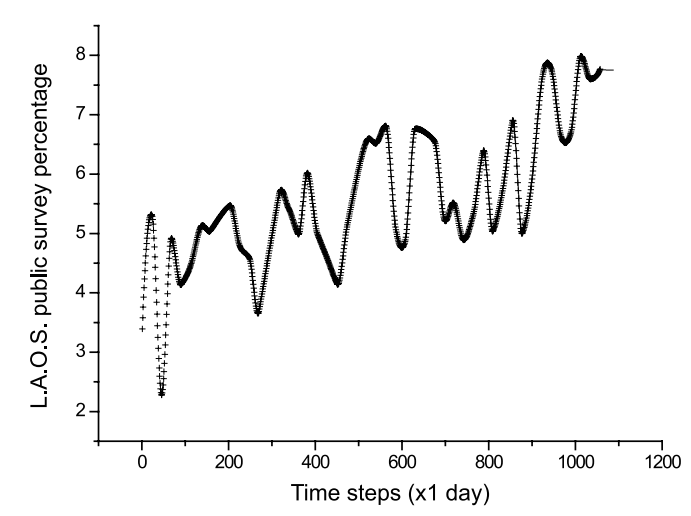

(e)

Figure 1. Actual (crosses) and predicted (solid line) time series for $n=30$ time steps ahead for ND(a), PASOK (b), KKE (c), SYRIZA (d), LAOS (e) political parties. The embedding dimension is $\mathrm{m}=6$.

In order to capture the polarization of voters we have decreased the degrees of freedom to 4 . We use as embedding dimension $m=4$ keeping the values of delay time the same from our previous article [1] and the number of near neighborhoods nn, as a rule of thumb [6] equal to $3 * m=12$, for all political parties. Actual and predicted time series for $\mathrm{k}=30$ time steps ahead are presented at Figs 2.(a), (b), (c), (d), (e) for ND, PASOK, KKE, SYRIZA, LAOS respectively.

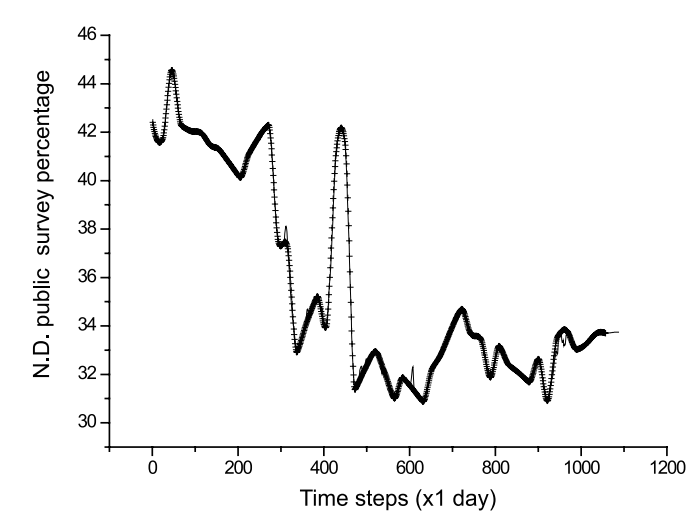

(a)

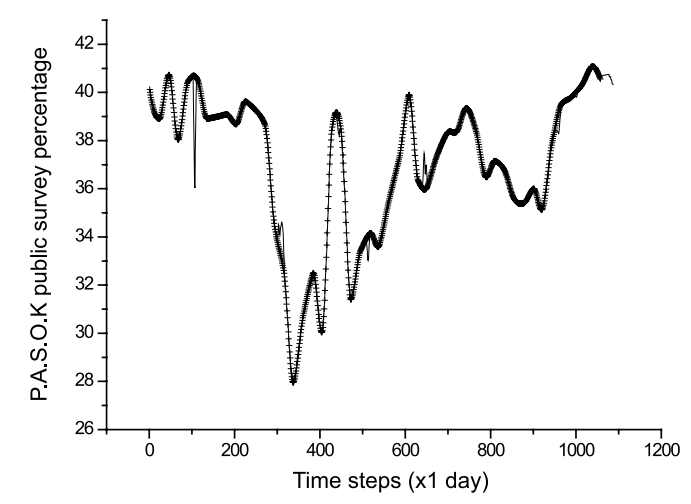

(b) 


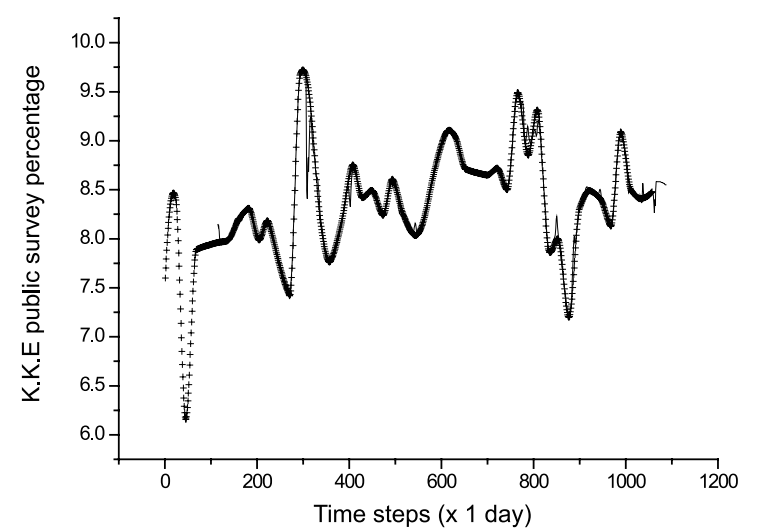

(c)

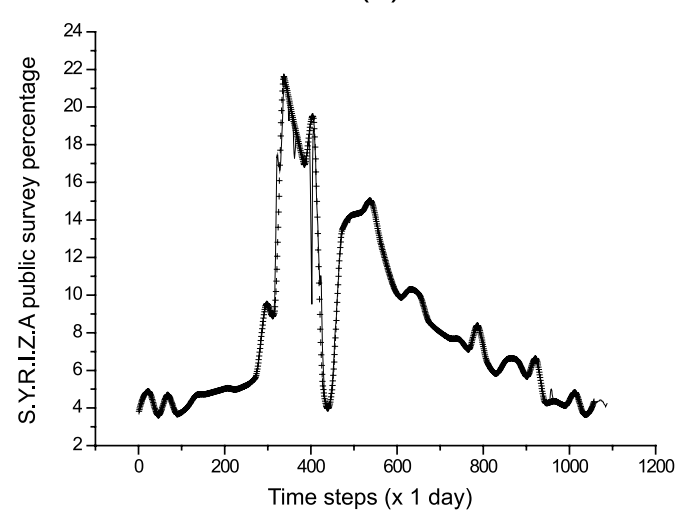

(d)

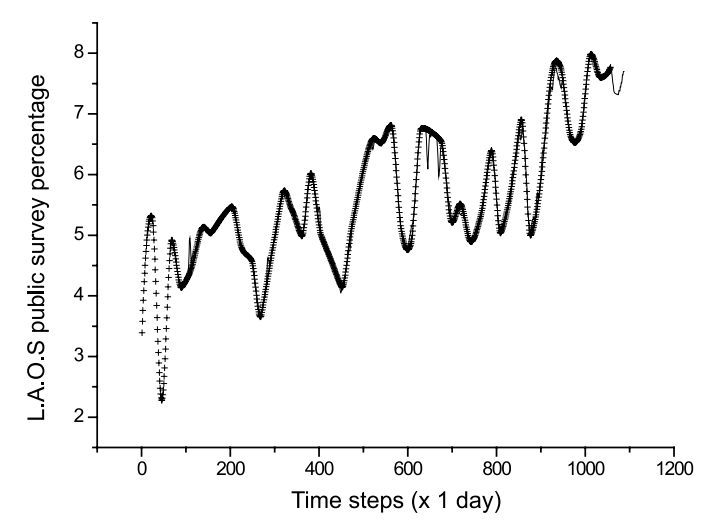

(e)

Figure 2. Actual (crosses) and predicted (solid line) time series for $n=30$ time steps ahead for ND (a), PASOK (b), KKE (c), SYRIZA (d), LAOS (e) political parties. The embedding dimension is $\mathrm{m}=4$.
At table 1 we present our out of sample estimation about political survey estimation for two embedding dimensions. The first is $\mathrm{m}=6$ and the second is $\mathrm{m}=4$. We estimate the mean error as $1.5 \%$ for ND and PASOK and $1 \%$ for the other political parties.

Table 1. Political survey estimation

\begin{tabular}{lcc}
\hline $\begin{array}{c}\text { Political } \\
\text { parties }\end{array}$ & $\begin{array}{c}\text { Embedding dimension } \\
\mathbf{m = 6} \\
\text { political survey } \\
\frac{\text { estimation \% }}{\text { for } \mathbf{4 / 1 0 / 2 0 0 9}}\end{array}$ & $\begin{array}{c}\text { Embedding dimension } \\
\mathbf{m = 4} \\
\text { political survey } \\
\frac{\text { estimation \% }}{\text { for } \mathbf{4 / 1 0 / 2 0 0 9}}\end{array}$ \\
\hline ND & 33.2 (Range $31.7-34.7$ ) & 34.0 (Range $32.5-35.5$ ) \\
PASOK & 39.0 (Range $37.5-40.5)$ & 41.1 (Range 39.6-42.6) \\
KKE & 8.6 (Range $7.6-9.6)$ & 8.6 (Range $7.6-9.6)$ \\
SYRIZA & 4.0 (Range $3.0-5.0)$ & 4.1 (Range $3.1-5.1$ ) \\
LAOS & 7.8 (Range $6.8-8.8)$ & 7.4 (Range $6.4-8.4)$ \\
\hline
\end{tabular}

The tables with predicted values can be found at appendix

\section{Conclusion}

In this paper, we applied a chaotic analysis to predict Greek political parties election results. From reconstruction of the systems' strange attractors, we achieved a 30 time steps out of sample prediction. The prediction with different degrees of freedom shown that, when the value of embedding dimension is 4 instead of 6 there is an increase of the percentage of larger political parties as N.D. and P.A.S.O.K. are. This can be interpreted as a polarization of voters which corresponds to a lesser degrees of freedom. The only exception is the percentage of SYRIZA political party. Even though the variation is too small and within mean error, we speculate that this behavior is due to that SYRIZA is composed of components that tend to come together in order to get more than $3 \%$.

\section{References}

1. M. P. Hanias, and L. Magafas, “Application of Physics Model in prediction of the Hellas Euro election results", JOURNAL OF ENGINEERING SCIENCE AND TECHNOLOGY REVIEW 2 (1), 104-112, (2009).

2. Miksovsky J., Raidl A., On some nonlinear methods of meteorological time series analysis, proceedings of WDS 2001 Conference (2007). Stam C. J., Pijn J. P. N., Pritchard W. S. Reliable detection of nonlin-earity in experimental time series with strong periodic component, Physica D, vol. 112, 361,(1998).

3. Hanias M.P., P.G.Curtis, J.E. Thallasinos, "Non-Linear Dynamics and Chaos: The case of the Price Indicator at the Athens Stock Ex-change", International Research Journal of Finance and Economics, Issue 11, pp.
154-163 (2007).

4. Hanias, P. Curtis and J. Thalassinos, "Prediction with Neural Net-works: The Athens Stock Exchange Price Indicator", European Journal of Economics, Finance And Administrative Sciences - Issue 9 (2007).

5. Hanias M. P. and D. A. Karras, "Efficient Non Linear Time Series Prediction Using Non Linear Signal Analysis and Neural Networks in Chaotic Diode Resonator Circuits", Springer Berlin / Heidelberg, Lecture Notes in Computer Science, Advances in Data Mining. Theoretical Aspects and Applications Volume 4597, pp 329-338 ( 2007).

6. Linsay PS, An efficient method of forecasting chaotic time series. Phys Lett A 1991;153:353-6. 


\section{Appendix}

\section{Prediction results with $\mathrm{m}=\mathbf{6}$}

Table 1. Out of sample predicted values of ND

\begin{tabular}{|c|c|}
\hline Time steps (x1 day) After 6-09-2009 & Percentage \% \\
\hline 1058 & 33.6932 \\
\hline 1059 & 33.6808 \\
\hline 1060 & 33.6662 \\
\hline 1061 & 33.6562 \\
\hline 1062 & 33.6518 \\
\hline 1063 & 33.6475 \\
\hline 1064 & 33.6445 \\
\hline 1065 & 33.6444 \\
\hline 1066 & 33.6432 \\
\hline 1067 & 33.6410 \\
\hline 1068 & 33.6419 \\
\hline 1069 & 33.6372 \\
\hline 1070 & 33.6354 \\
\hline 1071 & 33.6177 \\
\hline 1072 & 33.5863 \\
\hline 1073 & 33.5754 \\
\hline 1074 & 33.5623 \\
\hline 1075 & 33.5219 \\
\hline 1076 & 33.4901 \\
\hline 1077 & 33.4645 \\
\hline 1078 & 33.4083 \\
\hline 1079 & 33.3559 \\
\hline 1080 & 33.3015 \\
\hline 1081 & 33.2780 \\
\hline 1082 & 33.2425 \\
\hline 1083 & 33.2054 \\
\hline 1084 & 33.2248 \\
\hline $1085(4 / 10 / 2009)$ & 33.2014 \\
\hline 1086 & 33.2098 \\
\hline 1087 & 33.2131 \\
\hline
\end{tabular}

Table 2. Out of sample predicted values of PASOK

\begin{tabular}{|c|c|}
\hline Time steps (x1 day) After 6-09-2009 & Percentage \% \\
\hline 1058 & 40.5973 \\
\hline 1059 & 40.6261 \\
\hline 1060 & 40.6437 \\
\hline 1061 & 40.6556 \\
\hline 1062 & 40.6642 \\
\hline 1063 & 40.6708 \\
\hline 1064 & 40.6759 \\
\hline 1065 & 40.6800 \\
\hline 1066 & 40.6833 \\
\hline 1067 & 40.6861 \\
\hline 1068 & 40.6884 \\
\hline 1069 & 40.5275 \\
\hline 1070 & 40.3279 \\
\hline 1071 & 40.1000 \\
\hline 1072 & 39.8583 \\
\hline 1073 & 39.6164 \\
\hline 1074 & 39.3818 \\
\hline 1075 & 39.3532 \\
\hline 1076 & 39.1449 \\
\hline 1077 & 39.1309 \\
\hline 1078 & 39.1186 \\
\hline 1079 & 38.9486 \\
\hline 1080 & 38.9486 \\
\hline 1081 & 38.9487 \\
\hline 1082 & 38.9488 \\
\hline 1083 & 38.9489 \\
\hline 1084 & 38.9490 \\
\hline $1085(4 / 10 / 2009)$ & 39.0100 \\
\hline 1086 & 38.9521 \\
\hline 1087 & 38.9522 \\
\hline
\end{tabular}

Table 3. Out of sample predicted values of KKE

\begin{tabular}{|c|c|}
\hline Time steps (x1 day) After 6-09-2009 & Percentage $\%$ \\
\hline 1057 & 8.47156 \\
\hline 1058 & 8.47766 \\
\hline 1059 & 8.47632 \\
\hline 1060 & 8.47564 \\
\hline 1061 & 8.20717 \\
\hline 1062 & 8.03464 \\
\hline 1063 & 8.03475 \\
\hline 1064 & 8.03477 \\
\hline 1065 & 8.03589 \\
\hline 1066 & 8.03800 \\
\hline 1067 & 8.03803 \\
\hline 1068 & 8.04108 \\
\hline 1069 & 8.04110 \\
\hline 1070 & 8.04111 \\
\hline 1071 & 8.22112 \\
\hline 1072 & 8.59245 \\
\hline 1073 & 8.59248 \\
\hline 1074 & 8.59250 \\
\hline 1075 & 8.60088 \\
\hline 1076 & 8.60089 \\
\hline 1077 & 8.60090 \\
\hline 1078 & 8.60091 \\
\hline 1079 & 8.60092 \\
\hline 1080 & 8.60093 \\
\hline 1081 & 8.60529 \\
\hline 1082 & 8.60529 \\
\hline 1083 & 8.60529 \\
\hline 1084 & 8.60529 \\
\hline $1085(4 / 10 / 2009)$ & 8.60529 \\
\hline 1086 & 8.23031 \\
\hline 1087 & 8.04966 \\
\hline
\end{tabular}

Table 4. Out of sample predicted values of SYRIZA

\begin{tabular}{|c|c|}
\hline Time steps (x1 day) After 6-09-2009 & Percentage $\%$ \\
\hline 1058 & 4.27603 \\
\hline 1059 & 4.22362 \\
\hline 1060 & 4.19996 \\
\hline 1061 & 4.18198 \\
\hline 1062 & 4.18587 \\
\hline 1063 & 4.18109 \\
\hline 1064 & 4.17857 \\
\hline 1065 & 4.17733 \\
\hline 1066 & 4.19868 \\
\hline 1067 & 4.20022 \\
\hline 1068 & 4.20206 \\
\hline 1069 & 4.22889 \\
\hline 1070 & 4.23187 \\
\hline 1071 & 4.23447 \\
\hline 1072 & 4.23643 \\
\hline 1073 & 4.23738 \\
\hline 1074 & 4.23304 \\
\hline 1075 & 4.23141 \\
\hline 1076 & 4.22820 \\
\hline 1077 & 4.22332 \\
\hline 1078 & 4.21682 \\
\hline 1079 & 4.17960 \\
\hline 1080 & 4.17086 \\
\hline 1081 & 4.13286 \\
\hline 1082 & 4.12278 \\
\hline 1083 & 4.11223 \\
\hline 1084 & 4.07493 \\
\hline $1085(4 / 10 / 2009)$ & 4.04200 \\
\hline 1086 & 4.11756 \\
\hline 1087 & 4.17539 \\
\hline
\end{tabular}


Table 5. Out of sample predicted values of LAOS

\begin{tabular}{|c|c|}
\hline Time steps (x1 day) After 6-09-2009 & Percentage \% \\
\hline 1058 & 7.76382 \\
\hline 1059 & 7.76007 \\
\hline 1060 & 7.75815 \\
\hline 1061 & 7.75701 \\
\hline 1062 & 7.75627 \\
\hline 1063 & 7.75577 \\
\hline 1064 & 7.75542 \\
\hline 1065 & 7.75517 \\
\hline 1066 & 7.75497 \\
\hline 1067 & 7.75479 \\
\hline 1068 & 7.75462 \\
\hline 1069 & 7.75444 \\
\hline 1070 & 7.75424 \\
\hline 1071 & 7.75404 \\
\hline 1072 & 7.75383 \\
\hline 1073 & 7.75361 \\
\hline 1074 & 7.75340 \\
\hline 1075 & 7.75319 \\
\hline 1076 & 7.75300 \\
\hline 1077 & 7.75282 \\
\hline 1078 & 7.75267 \\
\hline 1079 & 7.75253 \\
\hline 1080 & 7.75241 \\
\hline 1081 & 7.75230 \\
\hline 1082 & 7.75222 \\
\hline 1083 & 7.75214 \\
\hline 1084 & 7.75208 \\
\hline $1085(4 / 10 / 2009)$ & 7.75203 \\
\hline 1086 & 7.75198 \\
\hline 1087 & 7.75195 \\
\hline
\end{tabular}

\section{Prediction results with $\mathrm{m}=4$}

Table 6. Out of sample predicted values of ND

\begin{tabular}{|c|c|}
\hline Time steps (x1 day) After 6-09-2009 & Percentage $\%$ \\
\hline 1058 & 33.7051 \\
\hline 1059 & 33.7093 \\
\hline 1060 & 33.7118 \\
\hline 1061 & 33.7134 \\
\hline 1062 & 33.7144 \\
\hline 1063 & 33.7151 \\
\hline 1064 & 33.7155 \\
\hline 1065 & 33.7159 \\
\hline 1066 & 33.7164 \\
\hline 1067 & 33.7173 \\
\hline 1068 & 33.7187 \\
\hline 1069 & 33.7206 \\
\hline 1070 & 33.7227 \\
\hline 1071 & 33.7250 \\
\hline 1072 & 33.7273 \\
\hline 1073 & 33.7293 \\
\hline 1074 & 33.7311 \\
\hline 1075 & 33.7326 \\
\hline 1076 & 33.7365 \\
\hline 1077 & 33.7395 \\
\hline 1078 & 33.7418 \\
\hline 1079 & 33.7433 \\
\hline 1080 & 33.7435 \\
\hline 1081 & 33.7441 \\
\hline 1082 & 33.7442 \\
\hline 1083 & 33.8441 \\
\hline 1084 & 33.0440 \\
\hline $1085(4 / 10 / 2009)$ & 34.0210 \\
\hline 1086 & 33.7431 \\
\hline 1087 & 33.7429 \\
\hline
\end{tabular}

Table 7. Out of sample predicted values of PASOK

\begin{tabular}{|c|c|}
\hline Time steps (x1 day) After 6-09-2009 & Percentage \% \\
\hline 1058 & 40.6014 \\
\hline 1059 & 40.6362 \\
\hline 1060 & 40.6591 \\
\hline 1061 & 40.6752 \\
\hline 1062 & 40.6871 \\
\hline 1063 & 40.6964 \\
\hline 1064 & 40.7038 \\
\hline 1065 & 40.7099 \\
\hline 1066 & 40.7148 \\
\hline 1067 & 40.7188 \\
\hline 1068 & 40.7222 \\
\hline 1069 & 40.7251 \\
\hline 1070 & 40.7277 \\
\hline 1071 & 40.7302 \\
\hline 1072 & 40.7326 \\
\hline 1073 & 40.7350 \\
\hline 1074 & 40.7373 \\
\hline 1075 & 40.7398 \\
\hline 1076 & 40.7424 \\
\hline 1077 & 40.7450 \\
\hline 1078 & 40.6517 \\
\hline 1079 & 40.6494 \\
\hline 1080 & 40.6468 \\
\hline 1081 & 40.6440 \\
\hline 1082 & 40.5246 \\
\hline 1083 & 41.5150 \\
\hline 1084 & 41.5054 \\
\hline $1085(4 / 10 / 2009)$ & 41.1400 \\
\hline 1086 & 41.3486 \\
\hline 1087 & 41.3345 \\
\hline
\end{tabular}

Table 8. Out of sample predicted values of KKE

\begin{tabular}{|c|c|}
\hline Time steps (x1 day) After 6-09-2009 & Percentage $\%$ \\
\hline 1058 & 8.46480 \\
\hline 1059 & 8.45107 \\
\hline 1060 & 8.44201 \\
\hline 1061 & 8.36346 \\
\hline 1062 & 8.26502 \\
\hline 1063 & 8.40075 \\
\hline 1064 & 8.33451 \\
\hline 1065 & 8.49595 \\
\hline 1066 & 8.58316 \\
\hline 1067 & 8.58394 \\
\hline 1068 & 8.58193 \\
\hline 1069 & 8.58199 \\
\hline 1070 & 8.58179 \\
\hline 1071 & 8.58150 \\
\hline 1072 & 8.58122 \\
\hline 1073 & 8.58097 \\
\hline 1074 & 8.58076 \\
\hline 1075 & 8.58058 \\
\hline 1076 & 8.58042 \\
\hline 1077 & 8.57530 \\
\hline 1078 & 8.57500 \\
\hline 1079 & 8.57462 \\
\hline 1080 & 8.57412 \\
\hline 1081 & 8.56672 \\
\hline 1082 & 8.56577 \\
\hline 1083 & 8.56463 \\
\hline 1084 & 8.56326 \\
\hline $1085(4 / 10 / 2009)$ & 8.55340 \\
\hline 1086 & 8.55132 \\
\hline 1087 & 8.54930 \\
\hline
\end{tabular}


Table 9. Out of sample predicted values of SYRIZA

\begin{tabular}{|c|c|}
\hline Time steps (x1 day) After 6-09-2009 & Percentage $\%$ \\
\hline 1058 & 4.29189 \\
\hline 1059 & 4.25894 \\
\hline 1060 & 4.26232 \\
\hline 1061 & 4.26044 \\
\hline 1062 & 4.29114 \\
\hline 1063 & 4.29753 \\
\hline 1064 & 4.30341 \\
\hline 1065 & 4.34795 \\
\hline 1066 & 4.35447 \\
\hline 1067 & 4.36044 \\
\hline 1068 & 4.36577 \\
\hline 1069 & 4.37030 \\
\hline 1070 & 4.41954 \\
\hline 1071 & 4.42308 \\
\hline 1072 & 4.42524 \\
\hline 1073 & 4.42605 \\
\hline 1074 & 4.37725 \\
\hline 1075 & 4.37429 \\
\hline 1076 & 4.36942 \\
\hline 1077 & 4.35928 \\
\hline 1078 & 4.30312 \\
\hline 1079 & 4.29436 \\
\hline 1080 & 4.23743 \\
\hline 1081 & 4.18433 \\
\hline 1082 & 4.17295 \\
\hline 1083 & 4.11939 \\
\hline 1084 & 4.07005 \\
\hline $1085(4 / 10 / 2009)$ & 4.11370 \\
\hline 1086 & 4.17437 \\
\hline 1087 & 4.23167 \\
\hline
\end{tabular}

The out of sample predicted values are shown at Table-10

Table 10. Out of sample predicted values of LAOS

\begin{tabular}{|c|c|}
\hline Time steps (x1 day) After 6-09-2009 & Percentage $\%$ \\
\hline 1058 & 7.75525 \\
\hline 1059 & 7.71735 \\
\hline 1060 & 7.66712 \\
\hline 1061 & 7.59912 \\
\hline 1062 & 7.51564 \\
\hline 1063 & 7.47506 \\
\hline 1064 & 7.38964 \\
\hline 1065 & 7.36555 \\
\hline 1066 & 7.35044 \\
\hline 1067 & 7.34044 \\
\hline 1068 & 7.33716 \\
\hline 1069 & 7.33166 \\
\hline 1070 & 7.32766 \\
\hline 1071 & 7.32460 \\
\hline 1072 & 7.32207 \\
\hline 1073 & 7.31979 \\
\hline 1074 & 7.32213 \\
\hline 1075 & 7.31917 \\
\hline 1076 & 7.38301 \\
\hline 1077 & 7.38162 \\
\hline 1078 & 7.38074 \\
\hline 1079 & 7.44708 \\
\hline 1080 & 7.44766 \\
\hline 1081 & 7.51058 \\
\hline 1082 & 7.41226 \\
\hline 1083 & 7.47757 \\
\hline 1084 & 7.64057 \\
\hline $1085(4 / 10 / 2009)$ & 7.44136 \\
\hline 1086 & 7.69940 \\
\hline 1087 & 7.69896 \\
\hline
\end{tabular}

